\title{
PSYCHO-SOMATIC DISORDERS AND PSYCHOLOGICAL MEDICINE
}

\author{
S. P. GHOSH \\ University of Delhi
}

\begin{abstract}
The author in the present investigation has studied twenty cases of psycho-somatic disorders and of three different categories: (i) digestive disorders, (ii) circulatory disorders, and (iii) cardiac disorders. He puts emphasis on the emotional stress as one of the important causative factors of these disorders. He also believes in the 'organ inferiority' hypothesis and family predesposition as other subsidiary factors.

He commends Zen and Yogic practices of Japan and India as effective therapies for these disorders. These practices like long practiced psycho-analysis have suggestive values and reform the whole personality of the patient.
\end{abstract}

\section{PART - I}

Mental disorders, at the time of Kraepelin, were considered as somatic disorders solely due to the pathological conditions of the brain. Kracpelin and his co-workers regarded mental disorders as somatic in origin, and treated them along the line of organic disorders. This somatogenic concept of Kraeplin was challenged by Freud and his followers; and they considered mental disorders as psycho-genic in origin. Both these somatogenic and psychogenic approaches were considered as one-sided; and the present day trend is to relate somatogenic and psycho-genic together, to study the psyche in soma and the sonza in psyche. The concept of psycho-somatic is based on close body-mind relationship and can better be explained with reference to Dr. Bose's Pansychic-psycho-physical parallelism. The somatic and psychic are two complementary processes and every disorder is at the same time a physiological and psychological one. Some disorders, however, for convenience are specifically called somatic or organic and others are psychic or functional and the difference between them is only a difference in degree. Psychological disorders, cspecially neuroses and psychoneuroses appear more frequently in a somatic guise than in a mental one. Patients suffering from constant headache, palpitation of heart, excessive sweating, indigestion, have been treated as suffering from physical diseases though no physical lesions have been demonstrated. Similarly patients suffering from rheumatism, weak heart, asthma, neuralgia, or neurities without any discoverable physical causes have been treated as persons suffering from physical disorders. Some patients complaining blindness, partial paralysis, symptoms of pseudo-pregnancy and hypersensibility of the skin, have been diagnosed as persons suffering from physical disorders without any reason for them. There are facts and phrases like 'nervous indigestion', 'Church diahrrhoea', 'stool neuroses', 'nervous agitation', 'hyperthyrodism'. The loss of appetite suffered by lovers, actors, singers and lecturers along with their stage fright is a fact usually known to all practising physicians.

There are the diseases in which physiological factors are predominating but they also show the presence of emotional states. These are psycho-neurotic symptoms and they improve very little by treatment along the physical lines; and physicians' failure arises from their ignorance of the emotional factors operating in these disorders. 
There are on the contrary, some psychological disorders like Paresis, Alcoholism and Sleeping Sickness whose origin has been traced back to the presence of physiological factors. Paretic patients for example suffer from psychotic symptoms which have their origin in the neuropsyphilitic infection of the organism. Chronic alcoholism is a well known psychotic disorder having its origin in the toxic effect of alcohol. Thus the psychotic disorders which are considered to be purely psychological in origin, show the presence of some physiological factors; and the ideal therapy for them will be one which will affect both the psychological and physiological aspects of the disorders. These disorders when treated by psychiatrists and psychoanalysists as purely psychic disorders they overlook the physiological basis of the disorders.

The psychoanalysist who treats Paresis by psychoanalysis alone ignores the somatic aspect of the disease and will surely be ridiculed. Thus mental disorders are neither somatic nor psychic but they are psycho-somatic. Psycho-somatics in wider sense though mean various types of mental disorders; but the cases studied in the present investigation fall under three categories (a) digestive disorders, (b) circulatory disorders, and (c) cardiac disorders. The total number of cases studied in this investigation was twenty. Out of the twenty cases, two cases were suffering from respiratory troubles, two from skin allergy and the remaining sixteen from intestinal troubles.

Case No.1.

The patient was a lady - aged about thirty three. She was married and had two children. She was admitted to the hospital for acute respiratory troubles. Her past history showed that upto thirty, she often suffered from skin allergy whenever she ate a particular type of fish-lobster. She had also mental upset twice-once before marrige at the age of eighteen and once after marriage. The recurrence of the allergy symptoms remained rather supressed for five years. The new troubles, the respiratory difficulties then started. The specialists in general medicine treated her with auto-vaxcine. The attack which was previously coming twice in a day was coming rather once in two days. Then she was removed from the hospital to the house and was treated by a yogi. Taking this yogic medicine, she became well. But we cannot say whether she has been cured permanently.

The observations on the intestinal cases demonstrates some common characteristics. Most of the cases had too much attachment for eating in earlier life. They were found to be chain smokers at the time of suffering. They were too much anxious for various types of medical examinations. Even some patients after the name of 'checking' wanted all sorts of examinations at their own cost. They displayed exhibitionistic tendencies. Some patients wanted more costly cabins though advantageous regarding nursing and treatment were the same. They wanted costly examination and costly treatment. They were not satisfied with free treatment of the hospital. Sense of guilt was also noted in some cases.

(i) Saul, Masserman and their co-workers have attributed all physiological disturbances to psycho-genic factors, This indced, is a significant contribution in the area of psychodynamics; but we have not sufficient materials to go so far. Though the mechanism of psycho-somatic illness is still obscure yet there are many findings which support the 
concept of psychogenic lesions. Psychic impulses, it has been observed, when intense and long sustained may cause anatomic damage of some digestive organs.

(ii) There is another group of workers who have tried to explain why a particular organ has been affected not another under the stress of emotional trauma or continued emotional tension. One plausible explanation is the 'organ selection' hypothesis which supports the view of organic defect of 'organ inferiority', as the reason for psycho-somatic diseases. The person who has suffered from dysentery from childhood is left with his bowels as more sensitive to emotional strain. Peculiar personality traits have also been noted in them. They are anxious, over-conscientious and aggressive. They, in one word, belong to obsessional type.

The predisposition for a certain disease runs in certain families. Most of the psychosomatic disorders are family affairs and attention should be given to the family to find out the genetic factors. The psycho-somatics inherit abnormally weak autonomic nervous system and their temperamental traits are inherited. Psycho-somatic disorders have also close linkage with socio-environmental factors. These disorders, are more common in urban population than in rural areas. They are more in higher social and occupational groups than in lower classes. Persons usually engaged in higher mental work are more liable to coronary thrombosis. The male suffers more from this group of disease than the female. Eskimos in Greenland have been found by a group of workers less susceptible to these diseases than western civilized man. Even among Eskimos those who have adopted western mode of life and thought, the disorders have been found to be more common in them. These facts demonstrate that psycho-somatic disorders are the diseases of civilization. (iii) Psycho-somatic disorders in a wider biological context have been emphasized by a third group of workers as biological protective devices. These disorders may arise if the individual be threatened with some danger and sustained these patterns of reactions unduly for a long time or as a way of life. The complex interplay of nervous mechanism in the individual's adaptation to life breaks down certain physical diseases, diseases of adaptation may result. Experiments on animal subjects, e.g., cats, dogs and sheep suggest that these animals under stress develop disorders of behavior with emotional and physical accompaniments having some resemblance to psycho-somatic disorders found in men.

(iv) Recently Franz Alexander and his co-workers have suggested that certain unconscious emotional conflicts tend to affect the functions of certain internal organs, producing specific vegetative responses and chronic functional disturbances. Now, of these various explanations, the 'presence of emotional stress' hypothesis support our findings. The presence of emotional stress was noted in all the cases, and the particular case cited here had mental breakdown twice in her life. The cases suffering from intestinal disorders, studied in this connection, suffered from stomach troubles from their childhood.

Concept of medicine: that which cures a disease is medicine - and this may be the root of a plat or a drop of the Ganges water or a costly drug like Chloromycitin. The concept of psycho-somatic medicine establishes a synthesis between organic and functional medicine. Now it is fully recognised that every medicine has got two counter parts - physical and psychological. The physical counterpart affects the body; and the psychological counterpart affects the mind. The psychological counterpart of the medicine lies in its suggestive value. 
The effects of suggestion, confidence and occultism of medicine develop in a patient 'the will for cure'. The presence of a family physician is an exmple in this respect. We run to the family physician whenever there is illness in the family because we have confidence in him. Once a Marwari patient refused to be operated by a veteran surgeon of Calcutta so long his family physician did not come and pointed out the portion of the stomach to be operated. The family physician was a layman in surgery, but still he had to locate the portion to be operated.

The contributions of Franz Alexander and his co-workers have permitted us to see the total problem of medicine from a better perspective. Modern medicine treats not the sick organ but the sick organism. Modern psychological medicine is more concemed with a sick personality rather than with the symptoms of sickness. Today even a successful general practitioner uses suggestion of a psychological nature to treat every form of disorder. The very 'will to get well', it has been recognised brings recovery in many organic diseases. Modern medicine is concerned with the physiological and the psychological aspects of all the diseases.

The following specific psycho-therapies may be useful in treating the psycho-somatic disorders:-

(i) Psycho-analysis of Freud and Jung may be effective therapies for treating disorders of this group. Freud's method of re-education by probing the unconscious traumatic experience of childhood; and the Jung's process of individualisation have been found to be successful therapies.

(ii) Zen therepy as practiced in Japan is an effective method for treating psychosomatic disorders. Zen claims to reform the whole personality of the patients in developing a new outlook in them. Zen speaks of 'Satori', which brings a sudden change in the individual. Satori experiences are something like illuminations of the West and yogic experiences of India.

(iii) Yogic practices in India have effective values for curing personality disorders. Chronic psycho-somatic disorders have also been reported to be cured by this method.

Now, whether we are using psycho-analysis, 'Zen-therapy' or yogic practiceseverywhere we see confidence and suggestion are playing an important role in curing these diseases.

MS. received IX 10,61 . 